Supporting Information

\title{
Protein folding dynamics as diffusion on a free energy surface: rate equation terms, transition paths and analysis of single-molecule photon trajectories
}

Nivin Mothi ${ }^{1,2}$ \& Victor Muñoz ${ }^{1,2,3, *}$

\begin{tabular}{|c|c|c|c|c|}
\hline $\begin{array}{c}\Delta G \\
\left(\mathrm{~kJ} \cdot \mathrm{mol}^{-1}\right)\end{array}$ & $\begin{array}{c}\log D \\
\left(X^{2} \cdot \mathrm{s}^{-1}\right)\end{array}$ & $\lambda_{1}\left(\mathrm{~s}^{-1}\right)$ & $\Delta t(\mathrm{~ns})$ & $\begin{array}{c}\text { Count-rate } \\
\left(\mathrm{ms}^{-1}\right)\end{array}$ \\
\hline 1 & 2.08 & 4,377 & 20 & 221 \\
\hline 3 & 2.08 & 3,648 & 20 & 221 \\
\hline 6 & 2.38 & 3,741 & 8 & 500 \\
\hline 9 & 2.68 & 3,288 & 3 & 1000 \\
\hline 15 & 2.78 & 620.5 & 1 & 1250 \\
\hline 20 & 2.91 & 148.4 & 0.5 & 1764 \\
\hline 25 & 3.00 & 30.2 & 0.2 & $* * * *$ \\
\hline
\end{tabular}

Table S1. Parameters used for the stochastic kinetic simulations of the free energy surfaces with barriers of different height, and for the simulations of time-stamped photon trajectories. $\phi=0.65$ was 
used for all simulations to change the barrier while keeping the exact same shape of the free energy surface. 

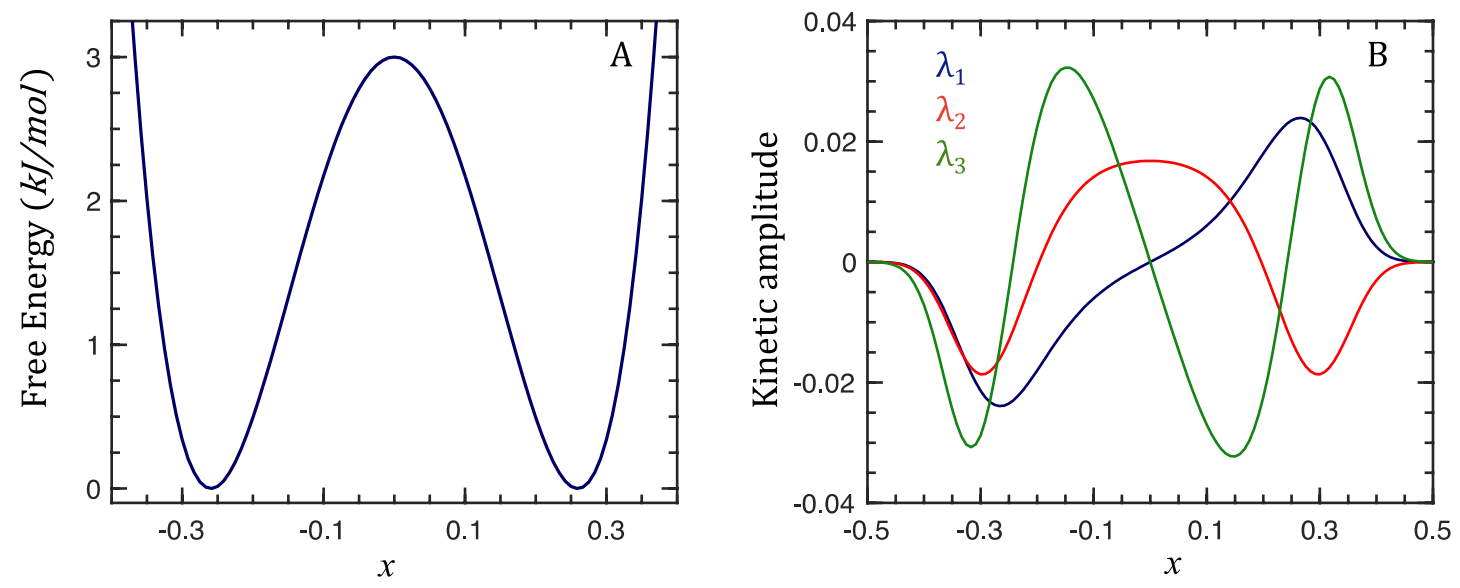

Figure S1. Free energy surface as a function of the order parameter $x(\mathrm{~A})$ and kinetic amplitudes of the three slowest non-zero eigenvalues obtained from the rate matrix (B). The slowest eigenvalue $\left(\lambda_{1}\right)$ corresponds to the overall kinetic relaxation between $F$ and $U . \lambda_{2}$ corresponds to the re-equilibration between the barrier top and the two minima (this eigenvalue thus represents the dynamics over the barrier, and its inverse is related to the average transition path time). $\lambda_{3}$ reflects the relaxation dynamics in both minima. Faster eigenvalues reflect increasingly localized dynamics along the free energy surface. 


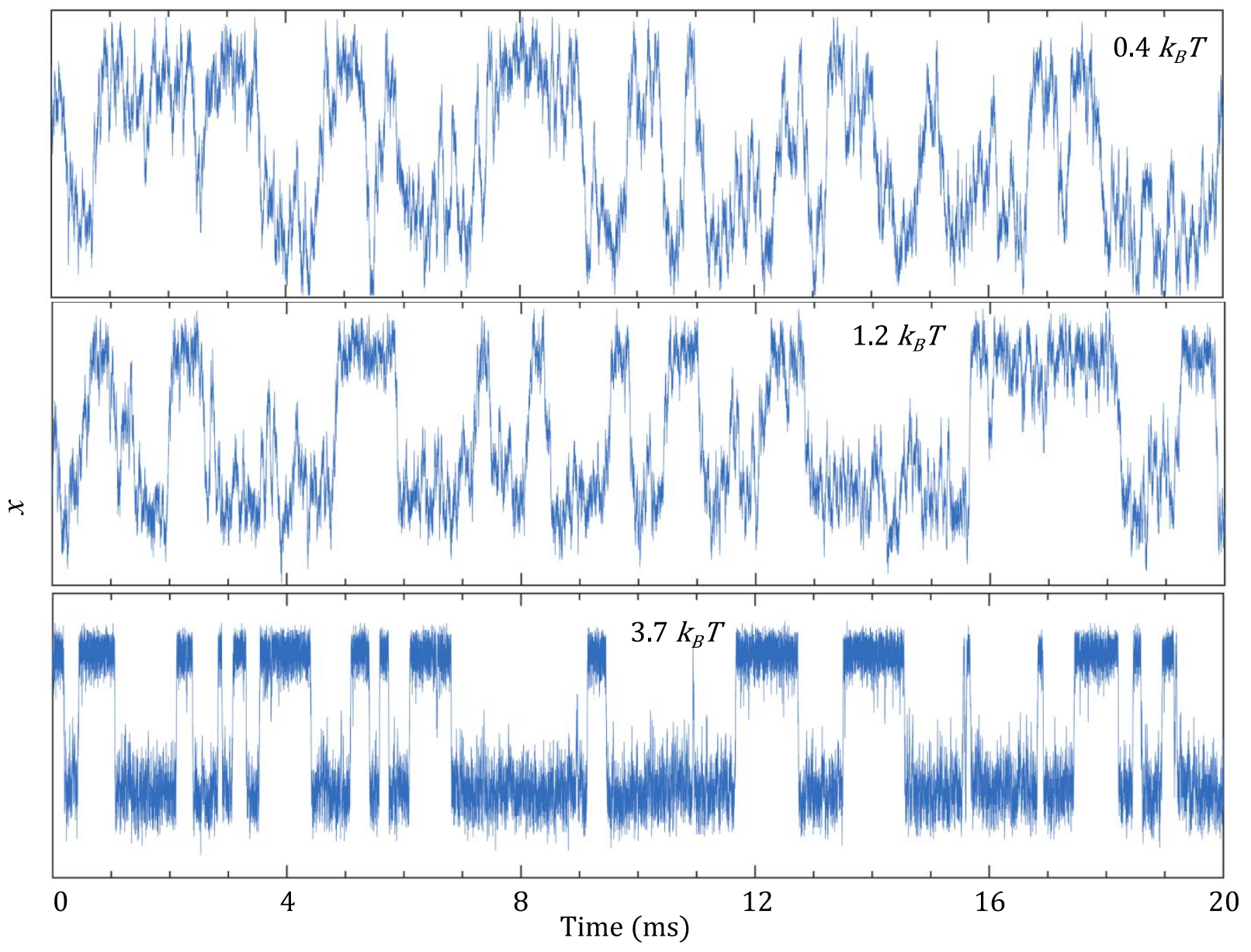

Figure S2. Examples of trajectory segments from the stochastic diffusive kinetic simulations on 1D free energy surfaces with different barrier heights. 

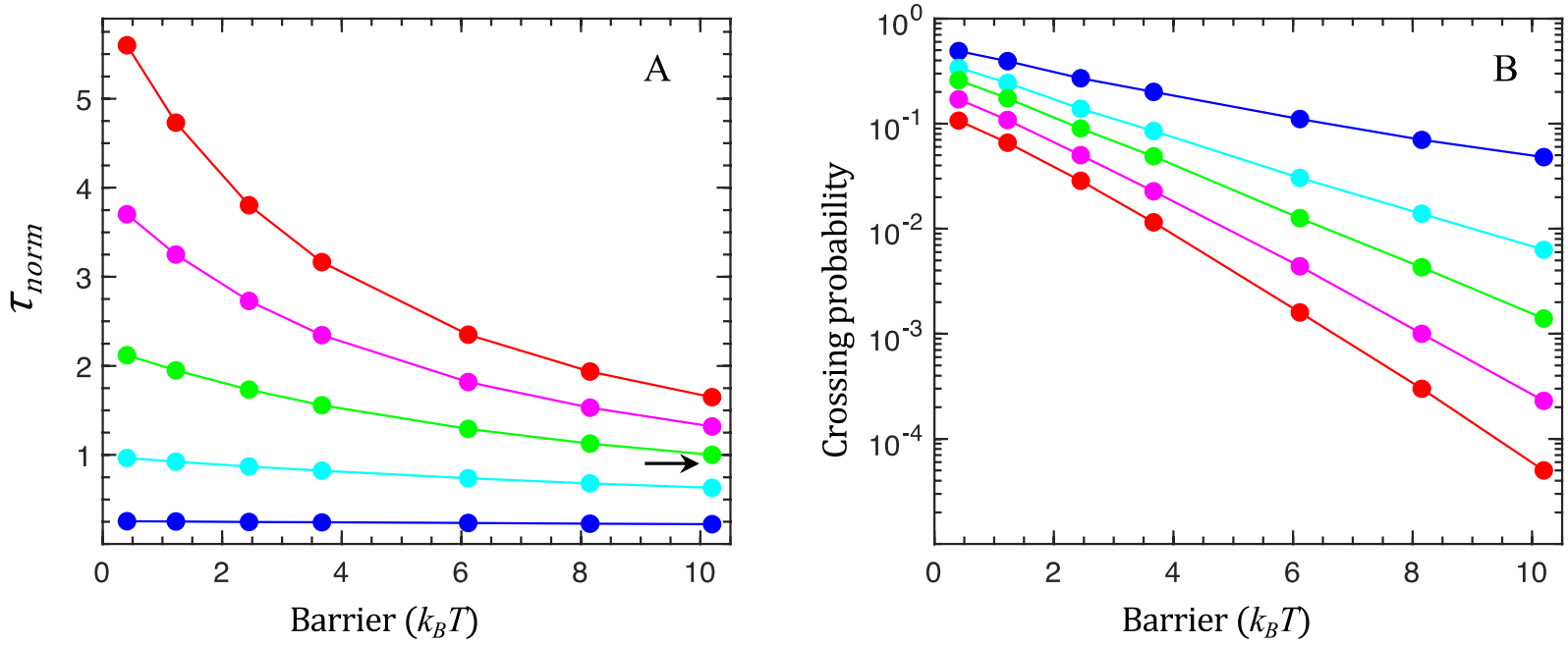

Figure S3. Transition path time and commitment probability for different folding scenarios. A) Transition path time (corrected for $D$ and normalized with respect to the data point indicated by the arrow) as a function of barrier for different definitions of transition region. Each datapoint represents the average of folding and unfolding transition path times. B) Commitment probability as a function of free energy barrier for different definitions of transition region. Color code follows Figure 2.

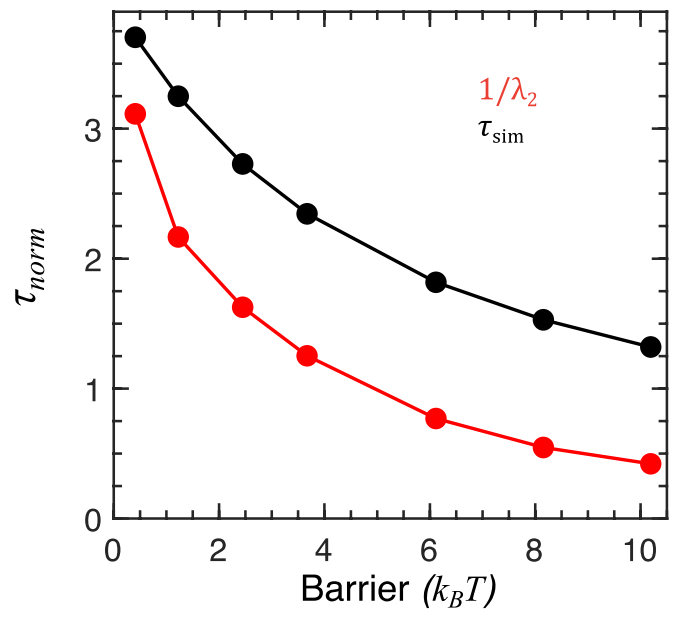


Figure S4. TPT for a transition region with $1 / 3$ boundaries (black), and the inverse of the second slowest non-zero eigenvalue (red) as a function of the barrier height. These values correspond to free energy surfaces with $\phi=0.65$, and are shown normalized as in Figure S2.
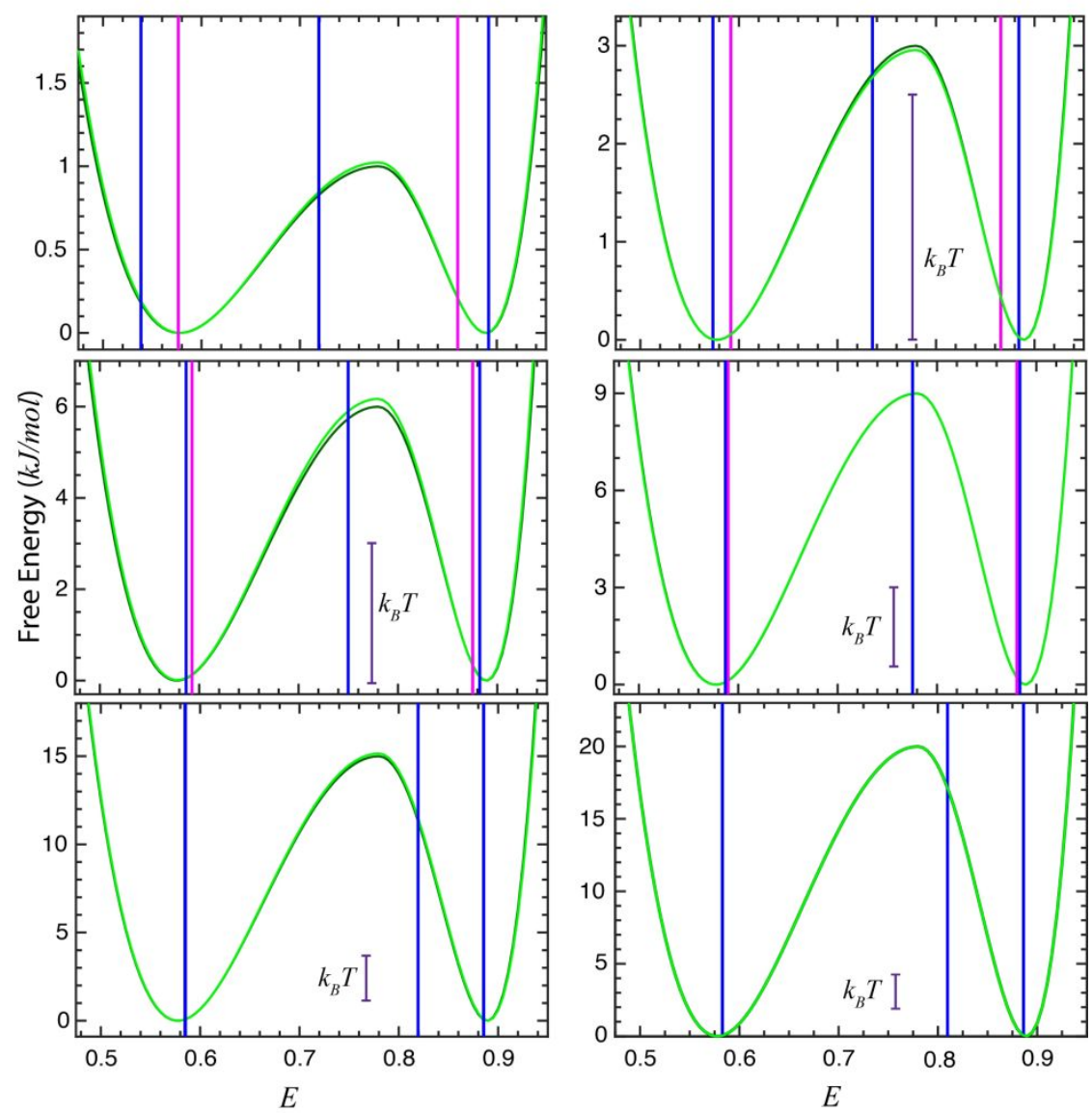

Figure S5. Free energy surfaces corresponding to different folding scenarios showing $E$ for the folded state $\left(\varepsilon_{F}\right)$ and unfolded states $\left(\varepsilon_{U}\right)$ obtained from the 2-state (magenta lines) and $\varepsilon_{F}, \varepsilon_{U}$ and $\varepsilon_{S}$ from the 3-state kinetic models (blue vertical lines). Light and dark green curves show the original FES used 
for the simulation and the FES retrieved from MLA analysis of the simulated photon trajectories, respectively. The panels are ordered in increasing barrier height from top left to bottom right. 